\title{
Study of Production of Green Conjugates of Silver Nanoparticles for Determination of Antimicrobial Potential against Biofilm Producing Staphylococcus aureus
}

\author{
Jaya Bharti ${ }^{1 *}$ and Abhishek Mathur ${ }^{2}$ \\ ${ }^{1}$ Department of Microbiology, Mewar University, Chittorgarh, Rajasthan, India \\ ${ }^{2}$ R\&D cum New product Development, EBEC-NCFT, New Delhi, India \\ *Corresponding author
}

\section{Keywords \\ Green synthesis, \\ Silver fused \\ Azadirachta indica \\ nanoparticles, \\ Biofilm, S. aureus, \\ Antimicrobial \\ activity.}

\section{Article Info}

Accepted:

21 June 2017

Available Online:

10 August 2017

\section{A B S T R A C T}

In recent era, Nanotechnology is a burning field for the researchers. Nanotechnology deals with the Nanoparticles having a size of 1-100 nm in one dimension used significantly concerning medical chemistry, atomic physics, and all other known fields. Nanoparticles are used immensely due to its small size, orientation, physical properties, which are reportedly shown to change the performance of any other material which is in contact with these tiny particles. These particles can be prepared easily by different chemical, physical, and biological approaches. But the biological approach is the most emerging approach of preparation, because, this method is easier than the other methods, eco-friendly and less time consuming. Staphylococcus aureus is a leading cause of nosocomial infections and the etiologic agent of a wide range of diseases associated with significant morbidity and mortality. Some of the diseases mediated by this species include endocarditis, osteomyelitis, toxic shock syndrome, food poisoning, and skin infections. Biofilms are well organized and complex aggregate of microorganisms, surrounded by a protective matrix of exo-polysaccharides and can adhere to each other on various surfaces. Because of increase in the drug resistance and the side effect of over use of antibiotic, it is necessary to find a suitable agent to reduce the growth of microorganism. Biofilm are known as a significant problem because biofilm formation protects pathogenic bacteria against antibiotics and is one of the main causes of development of chronic infections. Thus biofilm is the main cause of drug resistance in microbial strains. These strains cause pathogenicity to a maximum extent and thus cause resistance against the conventional antibiotics/drugs. The nanoparticles were synthesized by the concept of green synthesis by using the aqueous solution of Azadirachta indica leaf extract and silver nitrate. Silver was of a particular interest for this process due to its evocative physical and chemical properties. Staphylococcus aureus strains were isolated and screened for biofilm production. A fixed ratio of plant extract to metal ion was prepared and the color change was observed which proved the formation of nanoparticles. These nanoparticles were characterized by UV-Vis Spectrophotometer and SEM. The nanoparticles were found have the size ranges from 160-180 $\mathrm{nm}$. These fused Ag-Azadirachta indica* (Ag-AI*) nanoparticles were screened against biofilm producing strains of S. aureus. The results showed promising antimicrobial activity against these biofilm producers.

\section{Introduction}

Nanotechnology is a latest field of modern research dealing with synthesis, design and manipulation of nanosized particles.
Remarkable development in nanotechnology has opened a novel and very wide frontier of application that includes the synthesis of 
nanoscale material, exploration of their unique physical chemical and optoelectronic properties. Nanotechnology has gained tremendous importance in state of the art techniques for health care, food technology, cosmetics, environmental health, mechanics, optics, chemical industries, biomedical, electronics, space engineering, energy science, drug-gene delivery, optoelectronics, catalysis, light emitters, nonlinear optical devices, single electron transistors, and photo electrochemical applications etc (Colvin et al., 1994; Wang and Herron, 1991; Schmid, 1992; Mansur, 1995). In the present scenario, there is a growing interest in biological reduction of metal ions into metal nanoparticles particularly in the field of biology and medicine because of their distinct particle size and shape dependent properties (Sun et al., 2008; Ko, 2007). Bionanotechnology, a new approach, has emerged as the integration between biotechnology and nanotechnology for developing biosynthetic and environmentalfriendly technology for the synthesis of nanomaterials. Nanoparticles are of different size and shapes (Sun and Xia, 2002; Manna et al., 2000). These can be synthesized in different material compositions and surface modifications (Gref, 2003). The synthesized nanoparticles are found to exhibit size (Park, 2008) or shape dependent properties (Anker, 2008). The present study deals with the synthesis of silver nanoparticles using leaves extracts of Azadirachta indica (Neem). These nanoparticles were prepared were screened for antimicrobial activity against biofilm producing $S$. aureus.

\section{Materials and Methods}

\section{Preparation of leaf extracts of Azadirachta indica (Neem) leaves}

Fresh leaves of Azadirachta indica (Neem) were collected from the local garden. Leaves were thoroughly washed to remove the dust particles. Further, dried in the sun to remove moisture and then grinded to fine powder. $1 \%$ of Neem leaves were added to $250 \mathrm{ml}$ conical flask containing deionized water followed by stirring for 15 minutes followed by incubation for 30 minutes at $25^{\circ} \mathrm{C}$. Solution was centrifuge at $5000 \mathrm{rpm}$ for 30 minutes at $25^{\circ}$ C. Clear solution was collected followed by filtration (using 2.5 microns filter paper) with the help of vaccum pump. This solution was used for biosynthesis of silver nanoparticles or reducing the silver ions to metal silver forms.

\section{Synthesis of silver nanoparticles}

Concentration ratios of plant extract: $\mathrm{AgNO} 3$ was prepared i.e. 3:2 by varying the plant extract concentration and keeping the concentration of silver nitrate constant. $1 \mathrm{mM}$ silver nitrate was added to plant extract to prepare a final volume of $200 \mathrm{ml}$ of a solution. Typically, $10 \mathrm{~mL}$ of plant extract was added to $190 \mathrm{ml}$ of $1 \mathrm{mM}$ aqueous AgNO3. The change in the colour of solution was observed during the whole process while taking OD of the solution. The silver nanoparticles solution that was formed was centrifuged at 12,000 rpm for 15-20 minutes followed by dilution of pellet.

\section{$\mathrm{UV}-\mathrm{V}$ is spectral analysis}

This bioreduced aqueous component was taken to measure absorbance of the solution. U.V spectra of the ratio 3:2 showed the best results. The absorbance was determined from 200-700 nm.

\section{FT-IR spectral analysis}

The bioreduced silver nitrate solution (nanoparticles) was centrifuged at 12,000 rpm for 20 minutes and the pellet was collected and characterized using FT-IR (Bruker, Germany) in the range of $4000-400 \mathrm{~cm}^{-1}$ 


\section{SEM analysis of silver nanoparticles}

Morphology and size of the silver nanoparticles were investigated by SEM images at an accelerating voltage of $5 \mathrm{KV}$.

\section{Antimicrobial activity of silver nanoparticles against biofilm forming Staphylococcus aureus}

\section{Agar well diffusion method}

The agar well diffusion method was modified (Perez and Anesini, 1993). Nutrient agar medium (SCDM) was used for bacterial cultures. The culture medium was inoculated with Staphylococcus aureus (isolated locally) suspended in nutrient broth. A total of $8 \mathrm{~mm}$ diameter wells were punched into the agar and filled with silver nanoparticles. Standard antibiotic (Erythromycin $1 \mathrm{mg} / \mathrm{ml}$ ) were simultaneously used as the positive control. The plates were incubated at $37^{\circ} \mathrm{C}$ for $18 \mathrm{~h}$. The antibacterial activity was evaluated by measuring the diameter of zone of inhibition observed. The procedure for assaying antibacterial activity was performed in triplicates to confirm the readings of diameter of zone of inhibition observed for each of the test organism.

\section{Measurement of minimum inhibitory concentration (MIC) and Minimum bactericidal concentration (MBC)}

Silver nanoparticles were added in LB medium, respectively. Each bacterium culture Staphylococcus aureus were controlled at $10^{5}$ $10^{6} \mathrm{Cfu} / \mathrm{mL}$ and incubated at $37^{\circ} \mathrm{C}$. To establish the antimicrobial activity of nanoparticles on the bacterial growth, the minimum inhibitory concentration of nano particles for Staphylococcus aureus were determined by optical density of the bacterial culture solution containing different concentration of nano particles after $24 \mathrm{~h}$. All of the experiments (MIC) were triplicate, on three different days (Melaiye et al., 2005). The minimum bactericidal concentration (MBC), i.e., the lowest concentration of nanoparticles that kills $99.9 \%$ of the bacteria were also determined from the batch culture studies. For growth inhibitory concentration (PMIC) the presence of viable microorganisms were tested and the lowest concentration causing bactericidal effect were reported as MBC as suggested (Avadi et al., 2004). To test for bactericidal effect, a loopful from each flask were inoculated on nutrient agar and incubated at $35^{\circ} \mathrm{C}$ for $24 \mathrm{~h}$. The nanoparticles concentration causing bactericidal effect was selected based on absence of colonies on the agar plate.

\section{Results and Discussion}

In the present study, the silver nanoparticles were prepared by the green synthesis which was characterized by the change in color from pale yellow to brown as shown in figure 1 . Initially the solution colour was light yellowish than with time duration it turned from yellowish to light brown to dark brownish after 24 hours, after this no further colour change was observed. Colour change indicates the formation of silver nanoparticles. The sharp clear intense bands of silver nanoparticles were observed at 415 $\mathrm{nm}$ in case of 3:2 ratios (Figure 2). FT-IR spectrum was analyzed for identification of different biomolecules adsorbed on the surface of nanoparticles, and also to find out their role in reduction and stabilizing the nanoparticles. The FT-IR spectrum of synthesized silver nanoparticles shows strong bands at 3372.33, 2932.20, 2366.24, 2109.93, $1640.23,869.22,819.26$ and $779.09 \mathrm{~cm}^{-1}$. The peaks at $3372.33,2932.20$ and 1640.23 corresponds to $\mathrm{O}-\mathrm{H}$ groups, $\mathrm{H}$ bonded alcohols and phenols respectively. The peaks at $2366.24 \mathrm{~cm}^{-1}$ and 2109.93 corresponds to secondary amine. The spectral bands 
(1055.76-1413.76 $\mathrm{cm}^{-1}$ ) show presence of proteins which are responsible for the reduction of metal ions or affinity for metal nanoparticles. The bands $\left(779.09-869.22 \mathrm{~cm}^{-1}\right)$ show the presence of phytochemical constituents in the form of flavanoids/terpenoids. This illustrates that that silver ions in aqueous medium have been converted to nanosized elemental silver (Fig. $3)$.

Table.1 Antimicrobial activities of silver nanoparticles (AgNps) against S. aureus

\begin{tabular}{|c|c|c|}
\hline $\begin{array}{c}\text { Biofilm forming } \\
\text { pathogen }\end{array}$ & $\begin{array}{c}\text { Doses } \\
(\mathbf{p p m})\end{array}$ & $\begin{array}{c}\text { Diameter of zone of } \\
\text { inhibition (mm) }\end{array}$ \\
\hline \multirow{3}{*}{ S. aureus } & $\mathbf{5}$ & 00 \\
\cline { 2 - 3 } & $\mathbf{1 0}$ & 18 \\
\cline { 2 - 3 } & $\mathbf{2 0}$ & 35 \\
\hline
\end{tabular}

Table.2 MIC and MBC of silver nanoparticles (AgNps) against S. aureus

\begin{tabular}{|c|c|c|c|}
\hline \multirow{2}{*}{$\begin{array}{c}\text { Biofilm forming } \\
\text { pathogen }\end{array}$} & \multirow{2}{*}{$\begin{array}{l}\text { Doses } \\
\text { (ppm) }\end{array}$} & \multicolumn{2}{|c|}{ MIC/MBC (ppm) } \\
\hline & & MIC & MBC \\
\hline & 10 & 6.0 & 8.5 \\
\hline & 20 & 3.5 & 17.6 \\
\hline
\end{tabular}

Fig.1 Preparation of silver nanoparticles via green synthesis

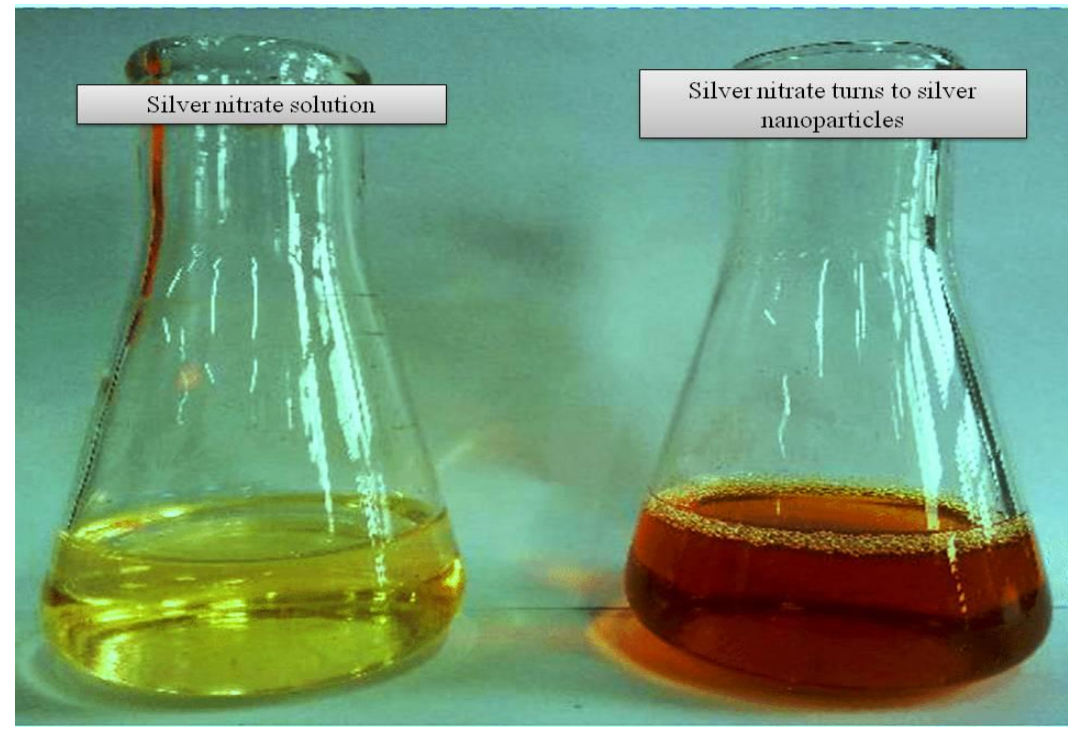


Fig.2 UV- absorption spectra of Ag nanoparticles

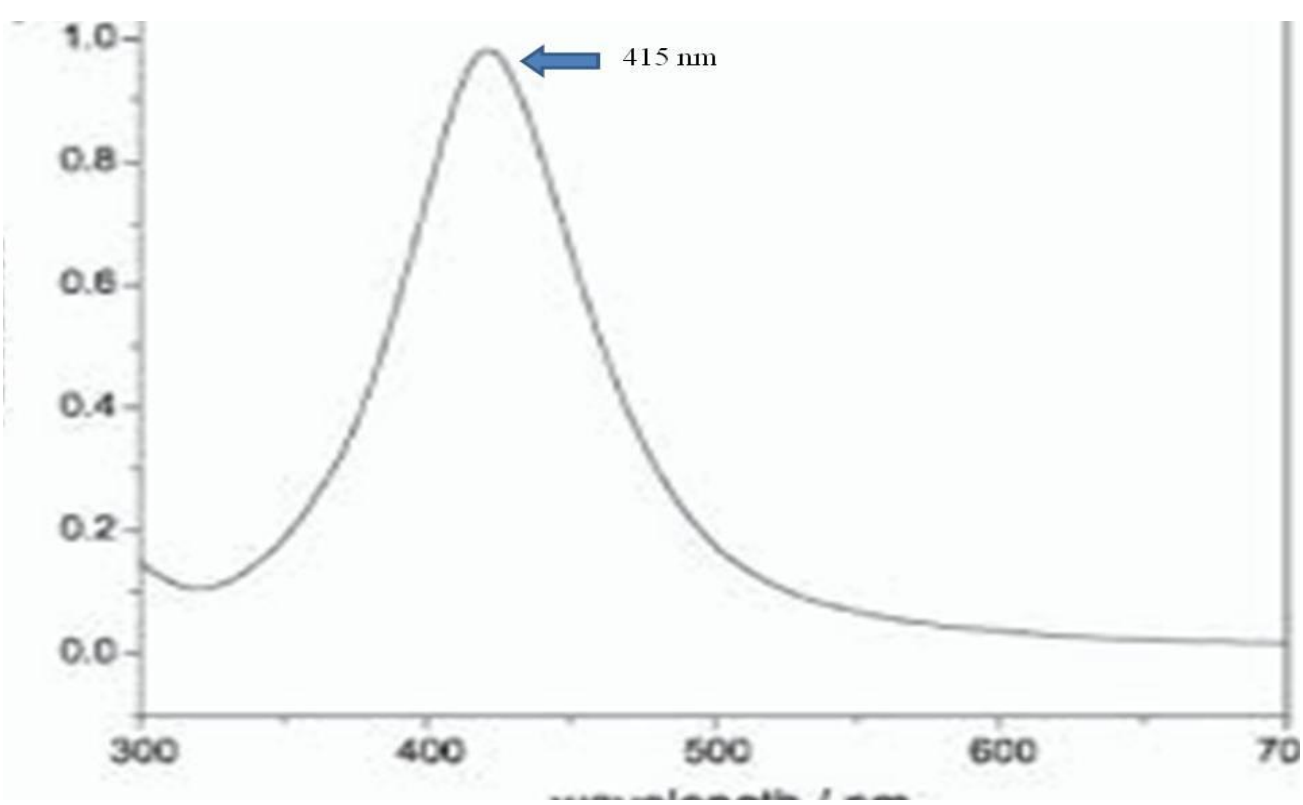

Fig.3 FT-IR spectra of silver nanoparticles prepared in 3:2 ratio (plant extract: $\mathrm{AgNO}_{3}$ )

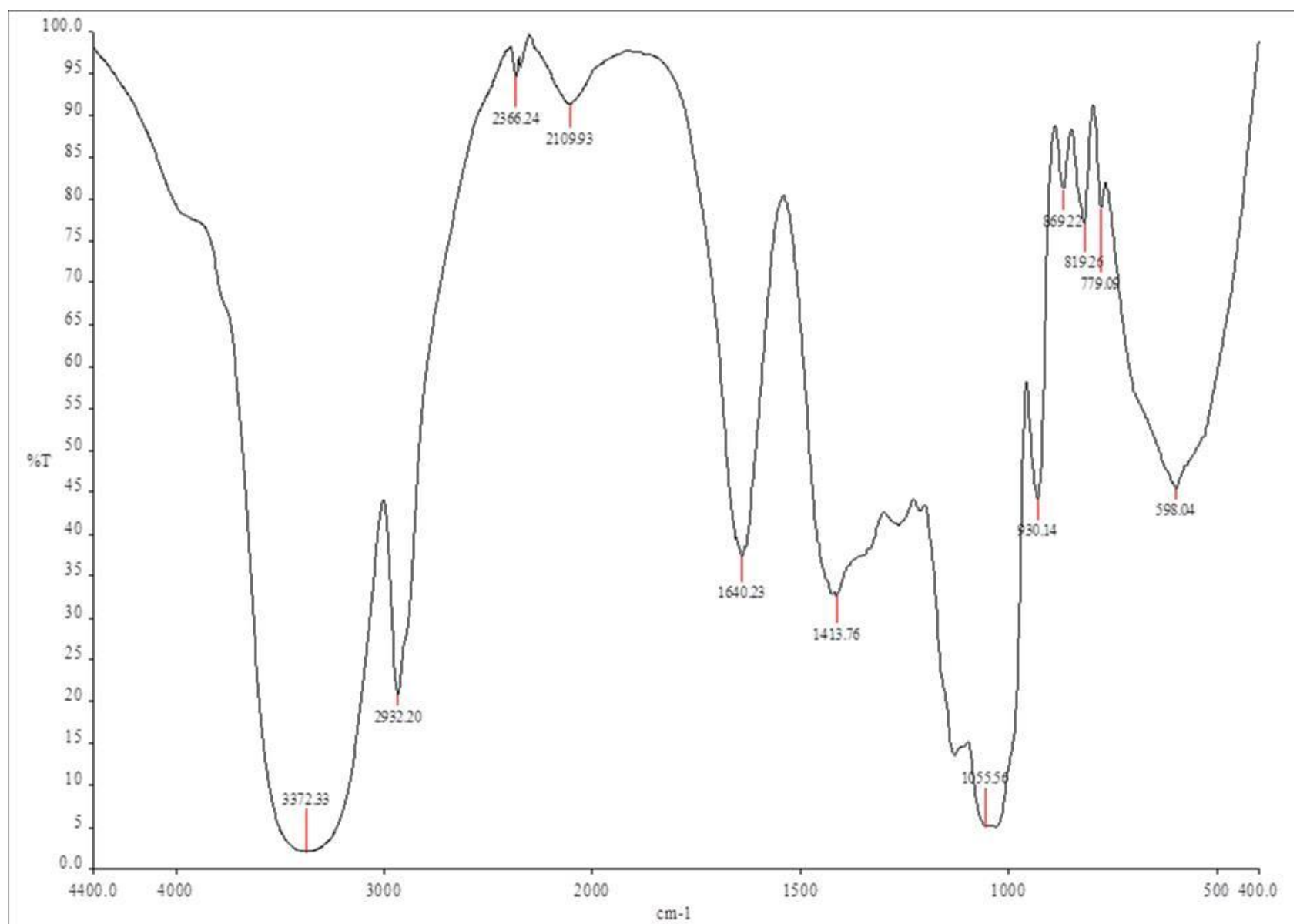


Fig.4 SEM image of silver nanoparticles prepared in 3:2 ratios (plant extract: $\mathrm{AgNO}_{3}$ )

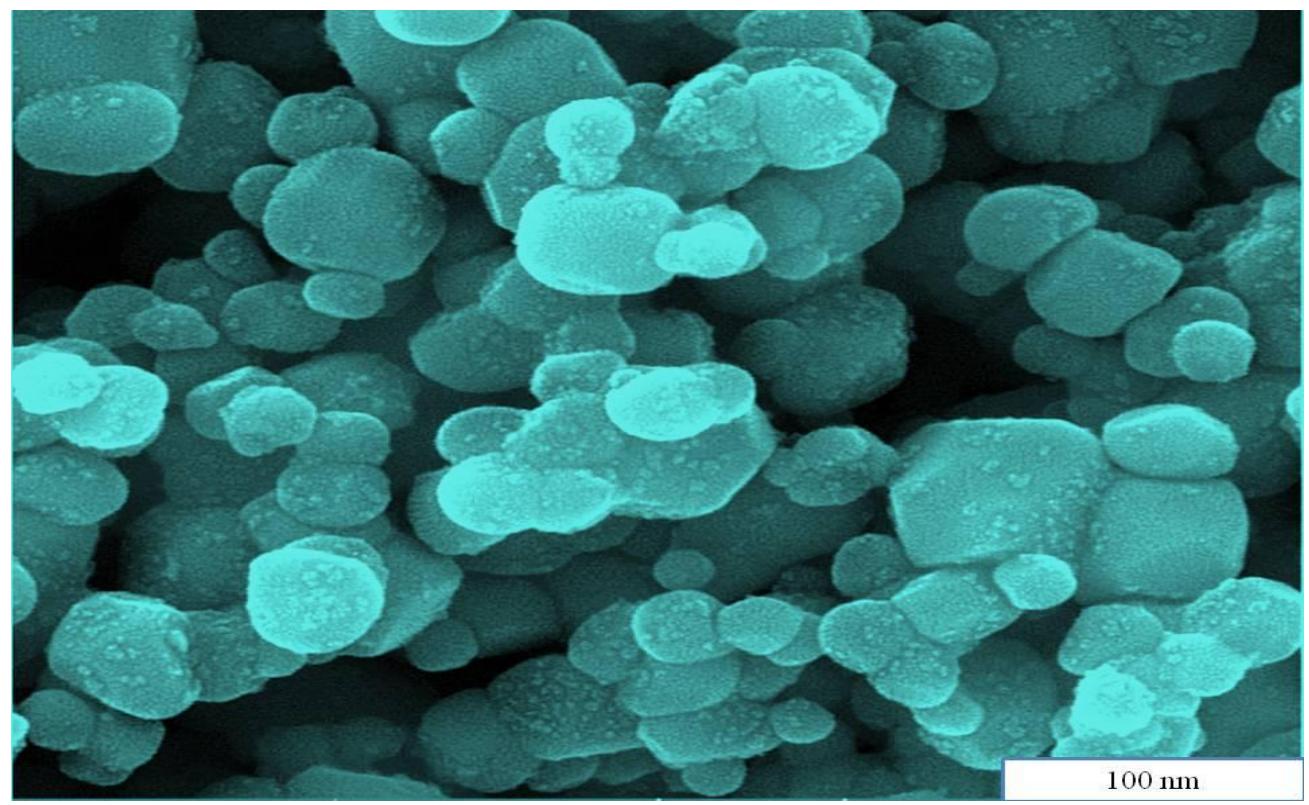

Fig.5 Antimicrobial activity of silver nanoparticles against biofilm producing $S$. aureus

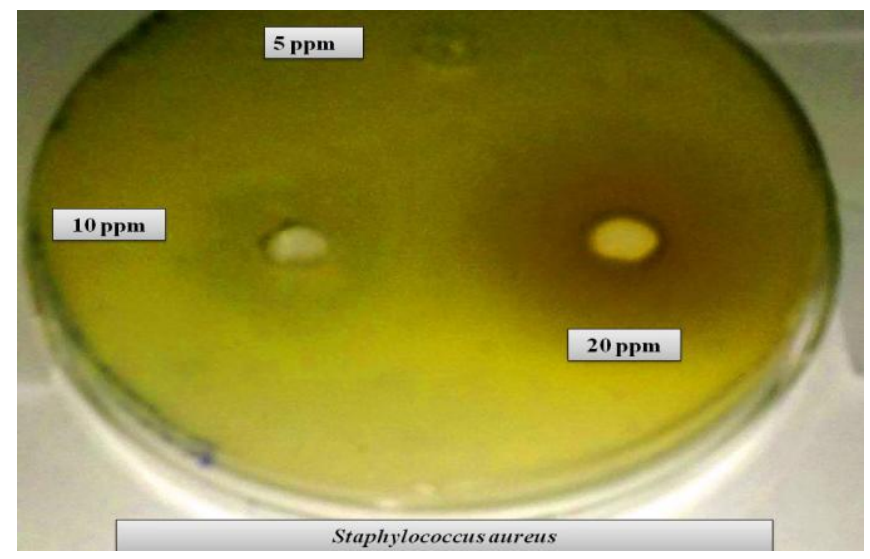

The nanoparticles prepared were of $100 \mathrm{~nm}$ size as determined by SEM (Figure 4). The antimicrobial activity of the nanoparticles synthesized was determined against isolated biofilm producing strain of $S$. aureus at 10,15 and $20 \mathrm{ppm}$. The results were found to be effective at 20 and $10 \mathrm{ppm}$ while at $5 \mathrm{ppm}$ there was no antimicrobial activity (Figure 5; Table 1). The results of MIC and MBC were also determined of the effective doses against the bacterium (Table 2). Silver nanoparticles were prepared by the use of different stabilizing agents viz. SDS and sodium citrate and were evaluated against biofilm producing S. aureus (Bharti et al., 2017). Trichoderma fused silver nanoparticles were evaluated for antifungal potential against phytopathogens (Gupta et al., 2016).

In conclusion, the present study suggests the antimicrobial significance of silver nanoparticles prepared by green synthesis. The nanoparticles are found to be the effective suitable agents for different pharmacological properties. From the present studies, it is clear that, minimum 
concentration of plant extract is suitable for preparation of green synthesis of silver nanoparticles. It is also concluded that, plant part(s) extracts containing secondary metabolites/active compounds are best capping agent for binding and providing a unique surface area to the nanoparticles. The results thus conclude that, silver nanoparticles synthesized by green chemistry are effective antimicrobial agents against $S$. aureus strains and thus can be utilized to formulate and effective antibacterial drug.

\section{References}

Anker, J.N. Biosensing with plasmonic nanosensors. Nature Materials, 7(6): 442453.

Avadi, M.R., Sadeghi, A.M.M., Tahzibi, A., Bayati, K., Pouladzadeh, M., Zohuriaan Mehr, M.J. 2004. Diethylmethyl chitosan as an antimicrobial agent: Synthesis, characterization and antibacterial effects. Eur. Polym., 40: 1355-1361.

Bharti, J., Mathur, A. 2017. Study of the antimicrobial effect of the silver nanoparticles against biofim producing Staphylococcus aureus strains. Int. J. Sci. Res. Pub., 7(3): 153-163.

Colvin, V.L., M.C. Schlamp, and A.P. Alivisatos. Light-emitting diodes made from cadmium selenide nanocrystals and a semiconducting polymer. Nature, 370(6488): 354-357.

Gref, R. 2003. Surface-engineered nanoparticles for multiple ligand coupling. Biomaterials, 24(24): 4529-4537.

Gupta, B.P., Raina, V., Jain, R., Mathur, A. 2016. Int. J. Pharma \& Bio Sci., Spl Ed. Int- BIONANO-2016, 33-41.

Ko. 2007. Direct nanoimprinting of metal nanoparticles for nanoscale electronics fabrication. Nano Lett., 7(7): 1869-1877.

Manna, L., E.C. Scher and A.P. Alivisatos. 2000. Synthesis of soluble and processable rod-, arrow-, teardrop-, and tetrapod-shaped CdSe nanocrystals. $J$. American Chem. Soc., 122(51): 1270012706.

Mansur, H.S. 1995. Photoelectrochemical properties of Q-state CdS particles in arachidic acid Langmuir Blodgett films. J. Chem. Society, Faraday Transactions, 91(4): p. 665-672.

Melaiye, A., Sun, Z., Hindi, K., Milsted, A., Ely, D., Reneker, D.H., Tessier, C.A,. Youngs, W.J. 2005. Silver(I)-imidazole cyclophane gem-diol complexes encapsulated by electrospun tecophilic nanofibers: formation of nanosilver particles and antimicrobial activity. J. Am. Chem. Soc., 127: 2285-2291.

Perez, C., Anesini, C. 1993. In vitro antimicrobial activity of Argentine folk medicinal plants against Salmonella typhii. J. Ethnopharmacol., 44: 41-46.

Schmid, G. 1992. Large clusters and colloids. Metals in the embryonic state. Chem. Rev., 92(8): 1709-1727.

Sun, Y. and Y. Xia. 2002. Shape-controlled synthesis of gold and silver nanoparticles. Sci., 298(5601): 2176-2179.

Sun, C., J.S.H. Lee and M. Zhang. 2008. Magnetic nanoparticles in MR imaging and drug delivery. Adv. Drug Delivery Rev., 60(11): 1252-1265.

Wang, Y. and N. Herron. 1991. Nanometersized semiconductor clusters: materials synthesis, quantum size effects, and photophysical properties. The J. Physical Chem., 95(2): 525-532.

\section{How to cite this article:}

Jaya Bharti and Abhishek Mathur. 2017. Study of Production of Green Conjugates of Silver Nanoparticles for Determination of Antimicrobial Potential against Biofilm Producing Staphylococcus aureus. Int.J.Curr.Microbiol.App.Sci. 6(8): 2280-2286. doi: https://doi.org/10.20546/ijcmas.2017.608.267 